Chapman University

Chapman University Digital Commons

Physical Therapy Faculty Articles and Research

Physical Therapy

9-20-2017

\title{
The Influence of Divided Attention on Walking Turns: Effects on Gait Control in Young Adults With and Without a History of Low Back Pain
}

Jo Armour Smith

Chapman University, josmith@chapman.edu

James Gordon

University of Southern California

Kornelia Kulig

University of Southern California

Follow this and additional works at: https://digitalcommons.chapman.edu/pt_articles

Part of the Other Rehabilitation and Therapy Commons, and the Physical Therapy Commons

\section{Recommended Citation}

Smith JA, Gordon J, Kulig K. The influence of divided attention on walking turns: Effects on gait control in young adults with and without a history of low back pain. Gait Posture. 2017;58:498-503. doi:10.1016/j.gaitpost.2017.09.019

This Article is brought to you for free and open access by the Physical Therapy at Chapman University Digital Commons. It has been accepted for inclusion in Physical Therapy Faculty Articles and Research by an authorized administrator of Chapman University Digital Commons. For more

information, please contact laughtin@chapman.edu. 


\title{
The Influence of Divided Attention on Walking Turns: Effects on Gait Control in Young Adults With and Without a History of Low Back Pain
}

\author{
Comments \\ NOTICE: this is the author's version of a work that was accepted for publication in Gait \& Posture. Changes \\ resulting from the publishing process, such as peer review, editing, corrections, structural formatting, and \\ other quality control mechanisms may not be reflected in this document. Changes may have been made to this \\ work since it was submitted for publication. A definitive version was subsequently published in Gait \& Posture, \\ volume 58, in 2017. DOI:10.1016/j.gaitpost.2017.09.019 \\ The Creative Commons license below applies only to this version of the article.
}

\section{Creative Commons License}

\section{(c) 1 (1) 90}

This work is licensed under a Creative Commons Attribution-Noncommercial-No Derivative Works 4.0 License.

\section{Copyright}

Elsevier 
The influence of divided attention on walking turns: effects on gait control in young adults with and without a history of low back pain.

\author{
Jo Armour Smith ${ }^{\mathrm{a}, \mathrm{b}}$, James Gordon ${ }^{\mathrm{b}}$, Kornelia Kulig
}

aDepartment of Physical Therapy, Harry and Diane Rinker Health Science Campus, Chapman University, 9401 Jeronimo Rd, Irvine, CA 92618, USA

${ }^{b}$ Division of Biokinesiology and Physical Therapy, University of Southern California, 1540 Alcazar Street, CHP-155, Los Angeles, CA 90089, USA

Corresponding author:

Jo Armour Smith

josmith@chapman.edu

\title{
$\underline{\text { Acknowledgements }}$
}

This work was supported in part by a New Investigator award from the Orthopaedic Section of the American Physical Therapy Association, a Student Grant-in-Aid award from the American Society of Biomechanics and a Florence P. Kendall Doctoral scholarship from the Foundation for Physical Therapy. 


\section{Abstract}

The cognitive control of gait is altered in individuals with low back pain, but it is unclear if this alteration persists between painful episodes. Locomotor perturbations such as walking turns may provide a sensitive measure of gait adaptation during divided attention in young adults. The purpose of this study was to investigate changes in gait during turns performed with divided attention, and to compare healthy young adults with asymptomatic individuals who have a history of recurrent low back pain (rLBP). Twenty-eight participants performed $90^{\circ}$ ipsilateral walking turns at a controlled speed of $1.5 \mathrm{~m} / \mathrm{s}$. During the divided attention condition they concurrently performed a verbal 2-back task. Step length and width, trunk-pelvis and hip excursion, inter-segmental coordination and stride-to-stride variability were quantified using motion capture. Mixed-model ANOVA were used to examine the effect of divided attention and group, and interaction effects on the selected variables. Step length variability decreased significantly with divided attention in the healthy group but not in the rLBP group (post-hoc $p=$ 0.024). Inter-segmental coordination variability was significantly decreased during divided attention (main effect of condition $p<0.000$ ). There were small but significant reductions in hip axial and sagittal motion across groups (main effect of condition $p=0.044$ and $p=0.040$ respectively), and a trend toward increased frontal motion in the rLBP group only (post-hoc $p=$ 0.048). These findings suggest that the ability to switch attentional resources during gait is altered in young adults with a history of rLBP, even between symptomatic episodes.

\section{Keywords}

Walking turn, divided attention, low back pain, variability 
Introduction

Functional, goal-oriented gait requires attention to navigate environmental obstacles. Even during steady-state walking, some attentional resources are utilized for gait [1]. This is demonstrated experimentally by cognitive-motor interference when gait is performed concurrently with another attention-demanding task. Cognitive-motor interference may be increased by a reduction in the automaticity of gait, an increase in the executive control resources utilized for steady-state gait [2], or as a result of impaired attentional capacity and processing[1]. Increased cognitive-motor interference during gait is evident in older adults and individuals with multiple clinical conditions including persistent pain. This is associated with impaired functional gait performance and increased risk of falls $[3,4]$.

The effect of cognitive-motor interference can be quantified at multiple levels of gait control. Spatiotemporal characteristics such as gait speed and step length provide information on control of overall task performance. Kinematic characteristics, such as coordination between segments (inter-segmental coordination) and individual joint excursions demonstrate how task performance is accomplished. In healthy adults, gait performance deteriorates during divided attention [5-9]. Existing evidence has demonstrated unchanged inter-segmental coordination [10], reduced [5] and unchanged lower-limb joint excursion [7] and reduced trunk excursion [11] during divided attention. However, as previous studies examined divided attention paradigms that involved additional mechanical demands, or did not control gait speed, the extent to which cognitive load alone accounts for these kinematic adaptations is unclear $[5,7,11]$.

Adaptable locomotor behavior is facilitated by stride-to-stride variability [12]. Healthy individuals demonstrate increased step length variability $[5,8]$, reduced step width variability [13], decreased variability in trunk-pelvis coordination [10] and decreased variability in trunk motion when attention is divided during steady-state gait [14]. Changes in variability during 
attention-demanding gait perturbations such as walking turns may provide further insight into cognitive-motor interference. Ipsilateral walking turns are changes in direction that occur toward the side of the stance limb. The reorientation into the new line of progression during ipsilateral turns may be accomplished within the stance phase of a single step (ipsilateral pivot strategy) and is achieved through rapid, axial rotation in the trunk, pelvis and hip $[15,16]$. As there is an immediate return to steady-state walking after the reorientation phase of the turn [15] the successful, consistent performance of the ipsilateral walking turn can be characterized by the length and width of the step immediately following the turn, and the variability of those parameters.

In people with low back pain (LBP), altered executive control of gait may result in an exaggerated response to divided attention [17]. Adults with chronic LBP demonstrate greater increases in stride length variability [17] and decreases in axial plane trunk-pelvic coordination variability [10] during divided attention compared with healthy controls. Existing studies have investigated middle-aged, symptomatic patients. In these individuals, executive function may be persistently impaired [18], or pain and fear of pain may demand additional attentional resources $[1,2,19]$. Many young individuals with persistent LBP have recurrent rather than chronic symptoms (rLBP)[20]. To better understand the mechanisms underlying cognitive-motor interference and LBP it is important to establish how individuals with rLBP respond to divided attention when they are asymptomatic. In particular, measures of trunk-pelvic inter-segmental coordination and joint excursion may provide valuable indices of cognitive-motor interference specific to the painful body region in this population.

The purpose of this study, therefore, was to compare the influence of divided attention on step length and width, inter-segmental coordination and joint excursion, and stride-to-stride variability during ipsilateral walking turns between asymptomatic young adults with a history of rLBP and healthy individuals. We hypothesized that in response to divided attention, all 
participants would demonstrate reduced step length and increased step width, increased step length/width variability, reduced trunk-pelvic and hip excursion and reduced trunk-pelvic coordination variability compared with baseline. We also hypothesized that these changes would be greater in the individuals with a history of rLBP.

\section{Methods}

$\underline{\text { Participants }}$

Twenty-eight young adults participated in the study (Table 1). Participants provided written informed consent. Individuals in the rLBP group were aged between 18 and 40 years [21] with at least a one-year history of rLBP and two functionally-limiting pain episodes exceeding 24 hours' duration in the preceding year [20] but were in symptom remission at the time of the data collection. Fear avoidance beliefs and impact of rLBP episodes were quantified using the Fear Avoidance Beliefs Questionnaire and the modified Oswestry Disability Index respectively $[22,23]$. Control participants (CTRL) had no history of low back pain and were individually matched to rLBP participants by sex, age, height, weight, and activity level.

\section{$\underline{\text { Instrumentation }}$}

Spatiotemporal and kinematic data were collected using an 11-camera motion capture system (200 Hz, Qualisys AB, Gothenburg, Sweden). Individual markers and marker clusters on the thorax (quantifying total trunk motion), pelvis, thighs, shanks and feet were used to define joint axes and track three-dimensional segment motion. Wireless force-sensitive resistor foot switches were attached bilaterally to the sole of participants' shoes under the heel and the first metatarsophalangeal joint $(3000 \mathrm{~Hz}$, TeleMyo DTS Telemetry, Noraxon USA Inc, Scottsdale, USA).

\section{Walking turns}

Participants performed multiple laps of a walking circuit that included straight walking and $90^{\circ}$ ipsilateral walking turns (Figure 1). Individuals with rLBP turned in the direction opposite 
the side of their predominant symptoms, and their matched control turned in the same direction. For both baseline (BASE) and divided attention (ATT) trials, participants performed the circuit at a controlled walking speed of $1.5 \mathrm{~m} / \mathrm{s} \pm 5 \%$. Average speed was quantified using photoelectric triggers and trials were repeated if the participant did not maintain the correct speed. All participants spontaneously utilized an ipsilateral pivot strategy to turn. $\underline{\text { Cognitive task }}$

For the ATT trials, participants performed a verbal 2-back task at the same time as the walking turns. An n-back task was selected as it requires continuous attention and does not utilize visual fixation or cause direct structural interference during walking[1]. The 2back version of the task was utilized as pilot testing demonstrated that participants found it challenging but were still able to perform the turns correctly at the controlled speed. Randomly generated strings of single digits were read to the participants at a rate of one approximately every two seconds. Participants responded "yes" when they heard a digit that was the same as one presented two digits earlier in the string. Baseline 2-back task performance was quantified during three trials in relaxed standing. During ATT trials, participants were instructed to prioritize the 2-back task over the walking turn, and were provided with feedback on the number of 2-back errors following each trial. As the duration of each trial was consistent for all participants, everyone received the same number of 2back stimuli.

\section{Data processing}

Marker trajectories were low-pass filtered at $10 \mathrm{~Hz}$. The stride cycle of each turn was determined using the voltage signals of the foot switches. Data were time-normalized within each stride cycle (Visual3D ${ }^{\mathrm{TM}}$ software, C-Motion Inc., MD, USA). Between 15 and 21 turns were analyzed for each participant for both BASE and ATT, as preliminary work indicated that a minimum of 15 trials provided stable stride-to-stride variability estimates.

Spatiotemporal variables and joint excursion 
Step length and step width (post-turn step, Figure 1) were calculated from the trajectories of the distal heel markers. For joint excursion, local coordinate systems for each segment were determined by a static calibration trial and peak-to-peak excursion of angular motion at the trunk-pelvis and hip (turn limb) was calculated across the turn stride cycle using Cardan angles [24]. The alignment of the trunk segment was normalized to the static standing trial to account for individual postural alignment [15]. Mean and standard deviation of the peak-to-peak amplitude of trunk-pelvic and hip motion was calculated for each participant and ensemble averages were calculated for the rLBP and CTRL groups during BASE and ATT trials.

$\underline{\text { Inter-segmental coordination }}$

Inter-segmental coordination between the trunk and the pelvis in the axial plane was quantified using the vector coding approach. This has been described in detail elsewhere [25] and we have demonstrated test-retest reliability of this approach [15]. Briefly, for each interval in the time series, a coupling angle between the segments was calculated. This coupling angle can be visualized as the angle from the right horizontal of a vector connecting successive points on an angle-angle plot of segment displacement. The mean coupling angle for each time interval across multiple trials for each participant was then calculated. From the mean coupling angle, the coordination pattern between the trunk and the pelvis at each time interval was then defined as inphase (trunk and pelvis rotating in the same direction), antiphase (trunk and pelvis rotating in opposite directions), pelvic phase (predominantly pelvic motion) and trunk phase (predominantly trunk motion) using 45 -degree bin widths $[26,27]$. The frequency that each coordination pattern occurred as a percentage of the total stride cycle was quantified for each participant for the BASE and ATT conditions (MATLAB ${ }^{\circledR}$, MathWorks, MA, USA).

\section{Stride-to-stride variability}


Within-subject stride-to-stride variability for step length and width and joint excursion was calculated as the standard deviation for the BASE and ATT turns in that individual. Stride-tostride variability of inter-segmental coordination was calculated using the mean angular deviation of the coupling angle across the stride cycle $[28,29]$. .

\section{$\underline{\text { Statistical analysis }}$}

Average numbers of 2-back errors per trial were compared between standing and the ATT condition, and between groups, using Wilcoxon signed ranks tests. Two-way mixed-model ANOVA were conducted for each variable to assess the main effect of divided attention (withinsubjects factor) and group (between-subjects factor) and any interaction effect. Variables that did not meet assumptions of normal distribution were log-transformed prior to statistical testing. Post-hoc comparisons for significant interaction effects were made using t-tests with a Bonferroni correction for number of tests performed within each factor $(\alpha=.05 / 2)$. Effect sizes for ANOVA main effects were calculated using partial eta squared $\left(\eta_{p}^{2}\right)$ and for post-hoc comparisons were calculated using Cohen's $d$. In both cases .8 indicates a large effect size, .5 a medium effect size and .2 a small effect size. Associations between changes in all variables during ATT were probed with Pearson correlation coefficients (SPSS ${ }^{\circledR}$ Version 21, IBM Corp., Armonk, NY).

\section{Results}

2-back task errors

The number of 2-back errors per trial at baseline in relaxed standing was the same in both groups (median \pm inter-quartile range, CTRL $0.3 \pm 0.2$, $\operatorname{LBBP} 0.3 \pm 0.3, p=0.465$ ). The number of errors during the ATT condition was the same as during relaxed standing $(p=0.904)$ and did not differ between groups (CTRL $0.3 \pm 0.3$, rLBP $0.4 \pm 0.5, p=0.348$ ).

$\underline{\text { Step length/width }}$ 
Step length and width were not significantly affected by divided attention in either group (Table 2).

Step length/width variability

The effect of divided attention on step length variability differed between groups (condition by group interaction; $F(\mathbf{1}, \mathbf{2 6})=4.86, p=0.037, \eta_{p}^{2}=0.157$, Table 2 ). Post-hoc testing indicated that there was no change in step length variability in the rLBP group $(p=0.634)$ but a decrease in variability in the CTRL group $(p=0.024, d=0.67$, Figure 2$)$. Step width variability was not significantly affected by divided attention in either group (Table 2). $\underline{\text { Joint excursion }}$

Trunk-pelvic motion did not differ with divided attention or between groups (Table 2). However, there was a significant main effect of condition on peak-to-peak amplitude of hip axial and sagittal plane excursion. Axial and sagittal hip motion was decreased in the divided attention condition $\left(\right.$ axial $\mathrm{F}(\mathbf{1}, \mathbf{2 6})=4.502, p=0.044, \eta_{p}^{2}=0.148$; sagittal $\mathrm{F}(\mathbf{1}, \mathbf{2 6})=4.661, p=$ $\left.0.040, \eta_{p}^{2}=0.152\right)$. There was a significant condition by group interaction for peak-to-peak amplitude of hip motion in the frontal plane $\left(F(\mathbf{1}, \mathbf{2 6})=7.233, \mathrm{p}=0.012, \eta_{p}^{2}=0.218\right.$ (Table 2). Post hoc comparisons indicated a trend toward a significant increase in motion in the rLBP group ( $p=0.048, d=0.55)$, with no change in the CTRL group $(p=0.122)$.

$\underline{\text { Joint excursion variability }}$

Stride-to-stride variability of trunk-pelvic excursion was not significantly affected by divided attention in the axial and sagittal planes (Table 2). In the frontal plane, stride-to-stride variability was decreased during divided attention in both groups $\left(F(\mathbf{1}, \mathbf{2 6})=7.328, p=0.012, \eta_{p}^{2}\right.$ $=0.220)$. Stride-to-stride variability of the hip was affected by divided attention. In the axial plane, there was a main effect of condition on stride-to-stride variability, with a decrease in axial plane variability during divided attention $\left(F(1,26)=9.054, p=0.006, \eta_{p}^{2}=0.258\right)$. Axial plane inter-segmental coordination 
There was no main or interaction effect for the frequency that each pattern of trunkpelvic inter-segmental coordination occurred across the stride cycle of the turn (Table 2). $\underline{\text { Inter-segmental coordination variability }}$

Mean coordination variability was lower during ATT than during BASE in both groups (main effect of condition on mean trunk-pelvic inter-segmental coordination variability; $F(\mathbf{1}, \mathbf{2 6})=$ 27.516, $\mathrm{p}<0.0001, \eta_{p}^{2}=0.934$, Figure 2).

$\underline{\text { Association between variables }}$

There was a significant positive correlation between change in step width and change in hip frontal plane excursion $(r=0.545, p=0.003)$. A larger increase in step width during ATT was associated with a larger increase in hip motion.

\section{Discussion}

This study investigated for the first time the effect of divided attention on gait during ipsilateral walking turns. Our data suggest that the impact of divided attention is affected by a history of rLBP, even between symptomatic episodes.

The performance of the walking turn was not affected by divided attention. However, in healthy individuals, the consistency of task performance significantly improved during divided attention. Substantial evidence indicates that motor performance improves when attention is redirected from an internal focus (on the movement itself) to an external focus [30]. This may be due to greater automaticity of task performance $[8,31]$. Similar redirection of attention may occur when individuals perform a motor task concurrently with a relatively simple cognitive task [32]. In the participants with a history of rLBP, there was no increased consistency in performance. This suggests that difficulty switching attentional focus may persist in individuals with LBP even between painful episodes and even when attentional resources are not being directed toward the experience of pain [19]. 
Divided attention did not affect the frequency that each pattern of trunk-pelvic intersegmental coordination occurred across the stride cycle of the turn. However, divided attention resulted in significantly decreased inter-segmental coordination variability across groups, with a large effect size. The findings from this study corroborate earlier work investigating the effect of divided attention on inter-segmental coordination variability between the trunk and the pelvis during steady-state treadmill walking in healthy adults [10]. The relationship between variability in task performance and coordination is complex. An optimal level of stride-to-stride coordination variability reflects adaptable use of degrees of freedom to ensure correct performance of the task goal under varying task conditions [33]. Therefore, too little intersegmental coordination variability may be associated with impaired task performance. However, in this study in healthy participants, consistency of walking turn performance improved even as coordination variability decreased, indicating that sufficient stride-to-stride variability was conserved during divided attention. In contrast to previous research in individuals with chronic LBP during steady-state walking [10], axial trunk-pelvic coordination variability was reduced equally in individuals with rLBP and healthy individuals during walking turns. This may be because sufficient variability in axial coordination is more essential for repeated successful walking turns than it is for steady-state gait due to the rapid modulation in the pattern of trunk-pelvic coordination that occurs during the turn [15]. This interpretation is consistent with previous evidence suggesting that the magnitude of variability in kinematic factors that are critical to successful gait performance is conserved in individuals with LBP during divided attention [34],

The effect of divided attention on joint excursion was less pronounced. Although there were significant decreases in axial and sagittal plane hip motion across groups, the effect sizes for these changes were small. In the rLBP group, divided attention resulted in greater hip frontal plane excursion. Examination of hip joint trajectories showed that this increase was a result of greater hip abduction during the swing phase of the turn-limb stride cycle. The significant 
positive correlation between increased hip excursion of the turn-limb and increased step width suggested that this may reflect a strategy in the rLBP group to enhance stability by increasing post-turn step width, and therefore increase their base of support immediately following the turn. Stride-to-stride variability of frontal trunk-pelvic excursion and hip axial excursion were also significantly reduced during the ATT trials, albeit with small effect sizes. However, in contrast to our hypotheses we did not observe that individuals with rLBP modified trunk-pelvic excursion differently than healthy individuals, and therefore these adaptations to divided attention do not seem to be influenced by a history of pain in that region.

The extent of cognitive-motor interference during divided attention is highly dependent upon the difficulty of both the cognitive and motor tasks. While gait performance may improve during divided attention with a less difficult cognitive task due to redirected attention, it may then deteriorate as cognitive load and resource competition increases [35]. It is difficult to directly compare the results of the present study with earlier work as the attention required to perform the walking turns at the correct speed is likely substantially larger than that required for steady-state treadmill walking. It is possible that utilizing a more difficult cognitive task, such as a 3-back task, would have elucidated greater group differences. In the present study it was clear that all participants effectively prioritized the 2-back task. This was indicated by no difference in number of 2-back errors during ATT compared with baseline. There was also no difference in the performance of the 2-back task between groups. Previous studies have demonstrated impaired cognitive performance in individuals with chronic pain $[10,18]$. These impairments appear to be more related to psychological distress than pain intensity [18]. The individuals in the rLBP group in this study had relatively low levels of fear avoidance and disability, and therefore they likely also had minimal psychological distress related to pain [22].

This study has important clinical implications that warrant further research. We have demonstrated that even in young adults with minimally disabling low back pain, and even 
when there is no pain at the time of the data collection, a history of rLBP is associated with altered allocation of attentional resources during perturbed gait. Determining the neural substrates of cognitive-motor interference during steady-state and perturbed walking using methodologies such as EEG or fNIRS will enhance the understanding of control mechanisms underlying altered gait in individuals with pain [36]. 
[1] Yogev-Seligmann G, Hausdorff JM, Giladi N. The role of executive function and attention in gait. Mov Disord 2008;23:329-42. doi:10.1002/mds.21720.

[2] Clark DJ. Automaticity of walking: functional significance, mechanisms, measurement and rehabilitation strategies. Front Hum Neurosci 2015;9. doi:10.3389/fnhum.2015.00246.

[3] Clark JE, Phillips SJ. A longitudinal study of intralimb coordination in the first year of independent walking: a dynamical systems analysis. Child Dev 1993;64:1143-57.

[4] Springer S, Giladi N, Peretz C, Yogev G, Simon ES, Hausdorff JM. Dual-tasking effects on gait variability: The role of aging, falls, and executive function. Mov Disord 2006;21:950-7. doi:10.1046/j.1532-5415.2002.50415.x.

[5] Lin M-IB, Lin K-H. Walking while Performing Working Memory Tasks Changes the Prefrontal Cortex Hemodynamic Activations and Gait Kinematics. Front Behav Neurosci 2016;10:715. doi:10.1016/S0966-6362(02)00190-X.

[6] Shkuratova N, Morris ME, Huxham F. Effects of age on balance control during walking. Archives of Physical Medicine and Rehabilitation 2004;85:582-8. doi:10.1016/j.apmr.2003.06.021.

[7] Agostini V, Fermo Lo F, Massazza G, Knaflitz M. Does texting while walking really affect gait in young adults? J NeuroEngineering Rehabil 2015:1-10. doi:10.1186/s12984-015-0079-4.

[8] Yogev-Seligmann G, Giladi N, Gruendlinger L, Hausdorff JM. The contribution of postural control and bilateral coordination to the impact of dual tasking on gait. Exp Brain Res 2013;226:81-93. doi:10.1007/s00221-013-3412-9.

[9] Al-Yahya E, Dawes H, Smith L, Dennis A, Howells K, Cockburn J. Cognitive motor interference while walking: A systematic review and meta-analysis. Neurosci Biobehav Rev 2011;35:715-28. doi:10.1016/j.neubiorev.2010.08.008.

[10] Lamoth CJ, Stins JF, Pont M, Kerckhoff F, Beek PJ. Effects of attention on the control of locomotion in individuals with chronic low back pain. J NeuroEngineering Rehabil 2008;5:13. doi:10.1186/1743-0003-5-13.

[11] Szturm T, Maharjan P, Marotta JJ, Shay B, Shrestha S, Sakhalkar V. The interacting effect of cognitive and motor task demands on performance of gait, balance and cognition in young adults. Gait Posture 2013;38:596-602. doi:10.1016/j.gaitpost.2013.02.004.

[12] Heiderscheit B, Hamill J, van Emmerik R. Variability of stride characteristics and joint coordination among individuals with unilateral patellofemoral pain. J Appl Biomech 2002;18:110-21.

[13] Grabiner MD, Troy KL. Attention demanding tasks during treadmill walking reduce step width variability in young adults. J NeuroEngineering Rehabil 2005;2:25. doi:10.1186/1743-0003-2-25.

[14] Dingwell JB, Robb RT, Troy KL, Grabiner MD. Effects of an attention demanding task on dynamic stability during treadmill walking. J NeuroEngineering Rehabil 2008;5:12. doi:10.1186/1743-0003-5-12.

[15] Smith JA, Kulig K. Trunk-pelvis coordination during turning: a cross sectional study of young adults with and without a history of low back pain. Clin Biomech 2016;12:58-64.

[16] Taylor MJD, Dabnichki P, Strike SC. A three-dimensional biomechanical comparison between turning strategies during the stance phase of walking. Hum Movement Sci 2005;24:558-73. doi:10.1016/j.humov.2005.07.005.

[17] Hamacher D, Hamacher D, Schega L. A cognitive dual task affects gait variability in 
patients suffering from chronic low back pain. Exp Brain Res 2014;232:3509-13. doi:10.1007/s00221-014-4039-1.

[18] Hart RP, Wade JB, Martelli MF. Cognitive impairment in patients with chronic pain: the significance of stress. Curr Pain Headache Rep 2003;7:116-26.

[19] Eccleston C, Crombez G. Pain demands attention: a cognitive-affective model of the interruptive function of pain. Psychol Bull 1999;125:356-66.

[20] Stanton TR, Latimer J, Maher CG, Hancock MJ. How do we define the condition "recurrent low back pain?" A systematic review. Eur Spine J 2009;19:533-9. doi:10.1007/s00586-009-1214-3.

[21] Hicks GE, Morone N, Weiner DK. Degenerative lumbar disc and facet disease in older adults: prevalence and clinical correlates. Spine 2009;34:1301-6.

doi:10.1097/BRS.0b013e3181a18263.

[22] George SZ, Valencia C, Beneciuk JM. A psychometric investigation of fear-avoidance model measures in patients with chronic low back pain. J Orthop Sports Phys Ther 2010;40:197-205. doi:10.2519/jospt.2010.3298.

[23] Fritz JM, Irrgang JJ. A comparison of a modified Oswestry low back pain disability questionnaire and the Quebec back pain disability scale. Phys Ther 2001;81:776-88.

[24] Schache AG, Blanch P, Rath D, Wrigley T, Bennell K. Three-dimensional angular kinematics of the lumbar spine and pelvis during running. Hum Movement Sci 2002;21:273-93.

[25] Needham R, Naemi R, Chockalingam N. Quantifying lumbar-pelvis coordination during gait using a modified vector coding technique. J Biomech 2014;47:1020-6. doi:10.1016/j.jbiomech.2013.12.032.

[26] Chang R, Van Emmerik R, Hamill J. Quantifying rearfoot-forefoot coordination in human walking. J Biomech 2008;41:3101-5. doi:10.1016/j.jbiomech.2008.07.024.

[27] Seay JF, Van Emmerik REA, Hamill J. Low back pain status affects pelvis-trunk coordination and variability during walking and running. Clin Biomech 2011;26:572-8. doi:10.1016/j.clinbiomech.2010.11.012.

[28] Hamill J, Haddad J. Issues in quantifying variability from a dynamical systems perspective. J Appl Biomech 2000;16:407-18.

[29] Miller RH, Chang R, Baird JL, Van Emmerik REA, Hamill J. Variability in kinematic coupling assessed by vector coding and continuous relative phase. J Biomech 2010;43:2554-60. doi:10.1016/j.jbiomech.2010.05.014.

[30] Wulf G. Attentional focus and motor learning: a review of 15 years. International Review of Sport and Exercise Psychology 2013;6:77-104.

doi:10.1016/j.psychsport.2009.01.006.

[31] Schaefer S, Jagenow D, Verrel J, Lindenberger U. The influence of cognitive load and walking speed on gait regularity in children and young adults. Gait Posture 2015;41:258-62. doi:10.1016/j.gaitpost.2014.10.013.

[32] Verrel J, Lövdén M, Schellenbach M, Schaefer S, Lindenberger U. Interacting effects of cognitive load and adult age on the regularity of whole-body motion during treadmill walking. Psychol Aging 2009;24:75-81. doi:10.1037/a0014272.

[33] Todorov E, Jordan MI. Optimal feedback control as a theory of motor coordination. Nat Neurosci 2002;5:1226-35. doi:10.1038/nn963.

[34] Hamacher D, Hamacher D, Herold F, Schega L. Are there differences in the dual-task walking variability of minimum toe clearance in chronic low back pain patients and healthy controls? Gait Posture 2016;49:97-101. doi:10.1016/j.gaitpost.2016.06.026.

[35] Lövdén M, Schaefer S, Pohlmeyer AE, Lindenberger U. Walking variability and workingmemory load in aging: a dual-process account relating cognitive control to motor control performance. J Gerontol B Psychol Sci Soc Sci 2008;63:P121-8.

[36] Hamacher D, Herold F, Wiegel P, Hamacher D, Schega L. Brain activity during walking: 
a systematic review. Neurosci Biobehav Rev 2015;57:310-27. doi:10.1016/j.neubiorev.2015.08.002.

Conflict of interest statement

The authors declare that there are no conflicts of interest associated with this work. 


\section{Figure legends}

Figure 1. Stride cycle of an ipsilateral walking turn to the right. Left: walking circuit, with events of ipsilateral turn stride cycle numbered and in black. Participants performed multiple laps of the walking circuit. In one corner of the circuit, participants performed a rapid $90^{\circ}$ ipsilateral walking turn before continuing to walk in the new line of progression. Right: stride cycle of turn demonstrating $1 \mathrm{a} \& \mathrm{~b}$ ) reorientation occurring during stance phase of the turn limb (right leg in this example), 2) initial contact of the contralateral limb (post-turn step) in new line of progression, and 3) final turn limb initial contact.

Figure 2. Left: individual data for changes in step length variability with divided attention showing significant decrease in variability in the CTRL group only (standard deviation, in meters. rLBP group $n=14$, CTRL group $n=14$ ) Right: individual data for changes in coordination variability with divided attention showing significant decrease in variability in both groups (angular deviation of coupling angle, in degrees. rLBP group $n=14, C T R L$ group $n=$ 14). 
Table 1. Participant demographics and clinical characteristics (median \pm inter-quartile range)

\begin{tabular}{llll}
\hline & CTRL $^{\mathrm{a}}$ & $\mathrm{rLBP}^{\mathrm{a}}$ & $\mathrm{p}$ \\
\hline Age (years) & $24.5 \pm 1.75$ & $26.5 \pm 4.75$ & .068 \\
Height (m) & $1.73 \pm 0.05$ & $1.73 \pm 0.09$ & .664 \\
Mass (kg) & $66.68 \pm 14.97$ & $67.70 \pm 23.42$ & .152 \\
$\begin{array}{l}\text { Time since first pain } \\
\text { episode (years) }\end{array}$ & $\mathrm{n} / \mathrm{a}$ & $5.8 \pm 4.2$ & $\mathrm{n} / \mathrm{a}$ \\
Baseline VAS (cm) & $\mathrm{n} / \mathrm{a}$ & $0.12 \pm 0.24$ & $\mathrm{n} / \mathrm{a}$ \\
FABQ & $\mathrm{n} / \mathrm{a}$ & $12.5 \pm 6.75$ & $\mathrm{n} / \mathrm{a}$ \\
ODI (\%) & $\mathrm{n} / \mathrm{a}$ & $18.0 \pm 15.0$ & $\mathrm{n} / \mathrm{a}$ \\
\hline $\mathrm{a} \mathrm{n}=14$ in each group, 8 women, 6 men ${ }^{\text {b }}$ physical activity sub-scale
\end{tabular}


Table 2. Summary of analyses of variance for all variables

\begin{tabular}{|c|c|c|c|c|c|c|}
\hline & \multicolumn{2}{|c|}{ Condition } & \multicolumn{2}{|l|}{ Group } & \multicolumn{2}{|c|}{$\begin{array}{l}\text { Group*condition } \\
\text { interaction }\end{array}$} \\
\hline & F ratio & $P$ & $\mathrm{~F}$ ratio & $P$ & F ratio & $P$ \\
\hline \multicolumn{7}{|l|}{ Gait performance } \\
\hline Step length & 2.90 & 0.10 & 0.13 & 0.72 & 2.43 & 0.13 \\
\hline Step width & 0.72 & 0.41 & 0.19 & 0.66 & 2.71 & 0.11 \\
\hline Step length variability & 2.39 & 0.13 & 0.51 & 0.48 & 4.86 & 0.04 \\
\hline Step width variability & 0.86 & 0.36 & 0.06 & 0.81 & 0.31 & 0.58 \\
\hline \multicolumn{7}{|l|}{ Inter-segmental coordination } \\
\hline$\%$ inphase coordination & 0.49 & 0.49 & 1.00 & 0.33 & 1.77 & 0.20 \\
\hline$\%$ antiphase coordination & 0.01 & 0.91 & 0.03 & 0.86 & 0.3 & 0.59 \\
\hline$\%$ trunk phase coordination & 0.98 & 0.33 & 3.23 & 0.08 & 2.05 & 0.16 \\
\hline$\%$ pelvic phase coordination & 0.98 & 0.34 & 0.05 & 0.93 & 0.72 & 0.41 \\
\hline Coordination variability & 27.52 & $<0.00$ & 1.48 & 0.24 & 0.06 & 0.82 \\
\hline \multicolumn{7}{|l|}{ Joint excursion } \\
\hline Trunk-pelvic axial & 0.54 & 0.47 & 1.92 & 0.18 & $<0.00$ & 0.96 \\
\hline Trunk-pelvic sagittal & 0.03 & 0.87 & 2.77 & 0.11 & 0.1 & 0.76 \\
\hline Trunk-pelvic frontal & 1.00 & 0.33 & 2.06 & 0.16 & 0.03 & 0.87 \\
\hline Trunk-pelvic axial variability & 3.62 & 0.07 & 0.45 & 0.51 & 0.05 & 0.34 \\
\hline Trunk-pelvic sagittal variability & 0.38 & 0.54 & 0.01 & 0.92 & 0.07 & 0.80 \\
\hline Trunk-pelvic frontal variability & 7.33 & 0.01 & 0.18 & 0.68 & 0.49 & 0.49 \\
\hline Hip axial & 4.50 & 0.04 & 0.51 & 0.48 & 0.98 & 0.33 \\
\hline Hip sagittal & 4.66 & 0.04 & 2.69 & 0.11 & 0.01 & 0.93 \\
\hline Hip frontal & 0.06 & 0.81 & 1.49 & 0.23 & 7.23 & 0.01 \\
\hline Hip axial variability & 9.05 & $<0.01$ & 0.23 & 0.64 & 0.01 & 0.93 \\
\hline Hip sagittal variability & 2.56 & 0.12 & 0.01 & 0.93 & 0.11 & 0.74 \\
\hline Hip frontal variability & 1.69 & 0.21 & 0.33 & 0.57 & 0.66 & 0.43 \\
\hline
\end{tabular}

Bold indicates significant effect 



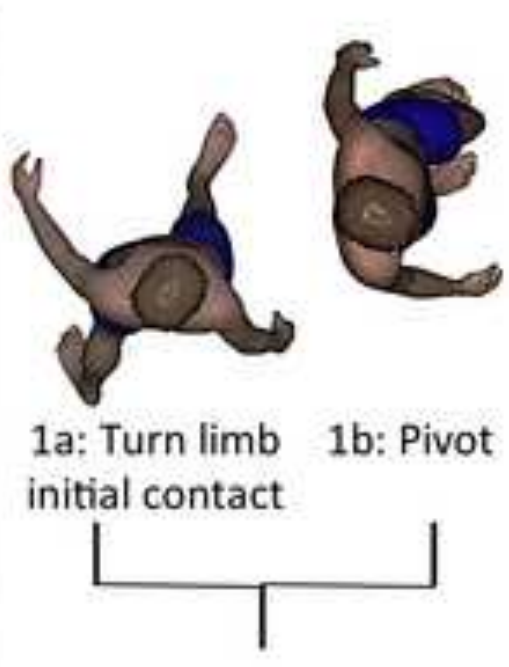

Reorientation phase

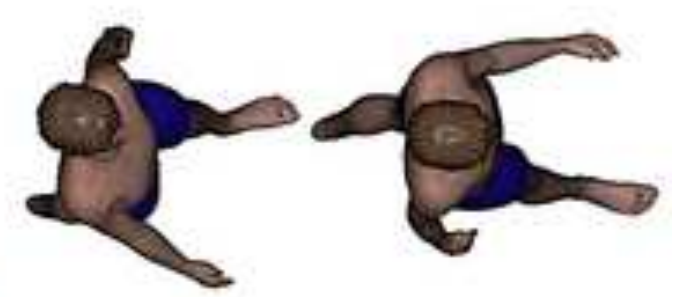

2: Contralateral initial contact

3: Turn limb initial contact

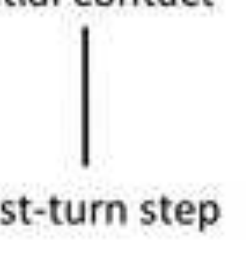



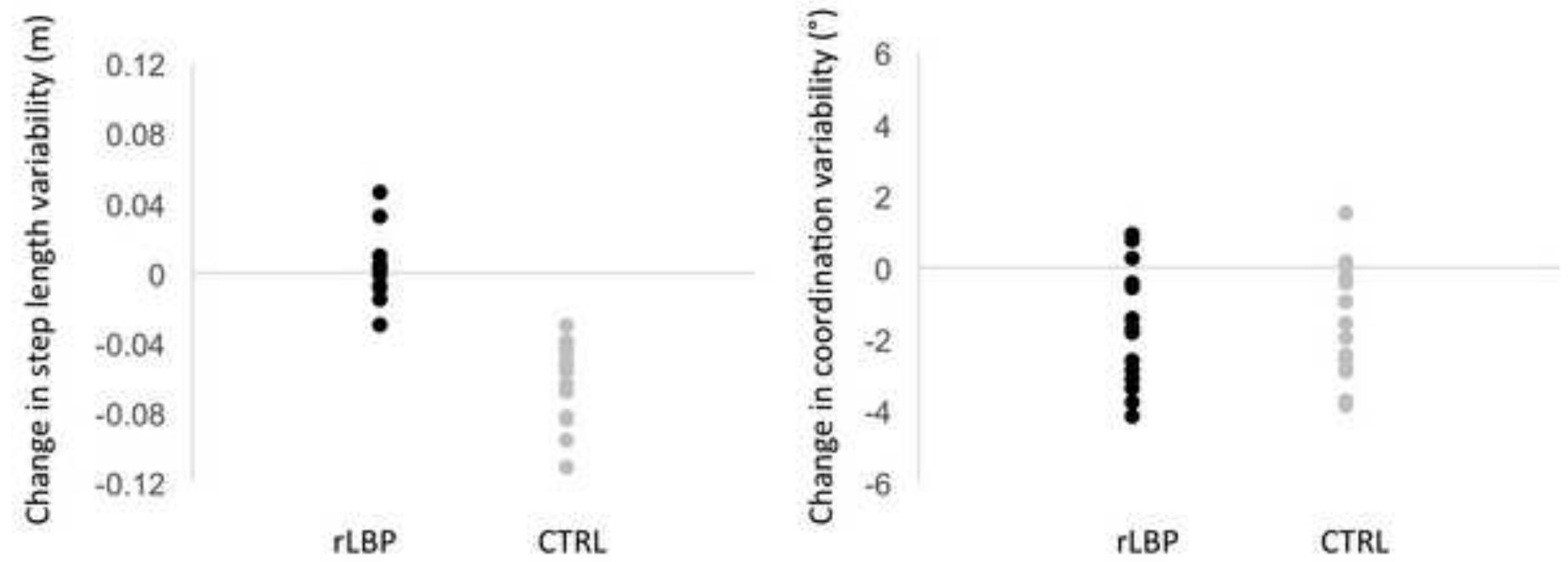
Research Highlights.

We investigated the effects of divided attention during walking turns

We compared healthy adults to asymptomatic adults with a history of low back pain

Turn performance consistency improved with divided attention in healthy adults only

Changes in cognitive control of gait persist between painful episodes 